\title{
Clinical Predictors of Prolonged Hospital Stay after Acute Stroke: Relevance of Medical Complications
}

\author{
Adrià Arboix $^{1,2^{*}}$, Joan Massons ${ }^{1}$, Luís García-Eroles ${ }^{3}$, Cecilia Targa ${ }^{1}$, Montserrat Oliveres ${ }^{1}$, \\ Emili Comes ${ }^{1}$ \\ ${ }^{1}$ Cerebrovascular Division, Department of Neurology, Capio-Hospital Universitari del Sagrat Cor, Universitat de Barcelona, \\ Barcelona, Spain; ${ }^{2}$ CIBER de Enfermedades Respiratorias, Instituto Carlos III, Madrid, Spain; ${ }^{3}$ Unit of Organization, Planning and \\ Information Systems, Consorci Sanitari del Maresme, Badalona, Spain. \\ Email: "aarboix@hscor.com,jmassonsc@terra.es, Igarciaer@csdm.es, 10021ctb@hotmail.com, 11466moi@comb.cat, \\ ecomes@hscor.com
}

Received August $18^{\text {th }}, 2012$; revised September $20^{\text {th }}, 2012$; accepted October $11^{\text {th }}, 2012$

\begin{abstract}
Purpose: This study aims to identify clinical predictors of prolonged hospital stay after acute stroke based on data collected from a prospective hospital-based acute stroke registry. Methods: All patients with first-ever ischemic stroke and primary intracerebral hemorrhage included in the Sagrat Cor Hospital of Barcelona stroke database over a 17-year period were assessed. Prolonged hospital stay was defined as hospitalization for longer than 12 days after admission. Demographic data, cardiovascular risk factors, clinical factors, neuroimaging findings, and outcome were compared in patients hospitalized for more or less than 12 days. Logistic regression analysis was used to assess the independent influence of statistically significant variables in the bivariate analysis and duration of hospitalization. Results: Of a total of 3112 acute stroke patients included in the study, prolonged hospital stay was recorded in 1536 (49.4\%). Male sex $(\mathrm{OR}=1.16)$, limb weakness $(\mathrm{OR}=1.79)$, vascular complications $(\mathrm{OR}=2.68)$, urinary complications $(\mathrm{OR}=2.56)$, and infectious complications $(\mathrm{OR}=1.78)$ were independently associated with longer stay, whereas symptom free at discharge $(\mathrm{OR}=0.45)$ and lacunar infarction $(\mathrm{OR}=0.43)$ were inversely associated with prolonged hospitalization. Conclusion: In-hospital medical complications (vascular, urinary, and infectious) are relevant factors influencing duration of hospitalization after acute stroke. Therefore, prevention of potentially modifiable risk factors for medical complications is an important aspect of the early management of patients with stroke.
\end{abstract}

Keywords: Medical Complications; Length of Hospital Stay; Stroke

\section{Introduction}

The length of stay is the major determinant of cost-determining factor during acute stroke hospitalization [1]. The length of stay may reflect the impact of the differences in health care system organization. Shortening the length of stay and using the bed and personal resources more efficiently are ways to achieve high patient turnover and, subsequently, provide more effective acute care for stroke patients $[1,2]$. Most studies concerning length of stay take into account the perspective of the administrators, are focused on costs, and do not necessarily consider the clinical relevance of findings [1]. Increased age, number of comorbidities and significant disability on discharge have been shown to influence length of stay in hospital in acute stroke patients [3,4]. Also, hospitals' characteristics especially the volume of treated patients and the organization services within the hospital may

${ }^{*}$ Corresponding author. play a key role [3]. A few studies, however, have identified complications during hospital stay as an independent factor associated prolonged hospital stay [5,6]. A Cochrane review concluded that appropriately resourced early supported discharge services can reduce the length of hospital stay as well as long term dependency and admission to institutional care [7].

\section{Purpose}

This study aims to identify clinical predictors of prolonged hospital stay after acute stroke in a large clinical series of stroke patients based on data collected from a prospective hospital-based acute stroke registry.

\section{Methods}

Between January 1986 and December 2002, data on 3420 patients with first-ever acute stroke admitted consecutively to the Department of Neurology of Sagrat Cor- 
Hospital of Barcelona, Spain, were collected prospectively in a stroke registry. Clinical data are entered in the database following a standardized protocol, the details of which have previously described [8]. Our institution is an acute care 350-bed teaching hospital in the city of Barcelona and serves a population of $\sim 250,000$ people. All patients with cerebrovascular disease are initially attended to in the emergency department and are then admitted to the Department of Neurology, which has 25 beds and a cerebrovascular division. Intensive care unit beds are also available. Patients are chosen for admission to the Department of Neurology if the reason for consultation is an acute cerebrovascular event occurring independently of the presence or absence of severe concomitant medical problems. The Cerebrovascular Study Group of the Spanish Neurological Society [9] was used for the classification of subtypes of stroke, which included transient ischemic attack, cerebral infarction (thrombotic, cardioembolic, lacunar, unusual etiology, and unknown cause), intracerebral hemorrhage, subarachnoid hemorrhage, spontaneous subdural hematoma, and spontaneous epidural hematoma. This classification is similar to that of the National Institute of Neurological Disorders and Stroke Classification [10]. Definitions of cerebrovascular risk factors have been used by our group in previous studies [11].

For the purpose of this study, data from patients with first-ever ischemic $(n=2704)$ and primary intracerebral hemorrhage $(n=408)$ attended over a 17 -year period were collected. Prolonged length of hospital stay was defined as 12 days or more. This cutpoint is based on the understanding that provision of acute stroke care, identification of stroke subtype, and preventing in-hospital complications is generally achieved in our Department of Neurology within the first 12 days of admission [8]. Prior to conducting the study, approval was obtained from the Ethical Committee of Clinical Research of the hospital.

All patients were admitted to the hospital within 48 hours of onset of symptoms. On admission, demographic characteristics; salient features of clinical and neurological examination and results of laboratory tests (blood cell count, biochemical profile, serum electrolytes, urinalysis); chest radiography; twelve-lead electrocardiography; and brain CT and/or MRI were recorded. Other investigations, including angio-MRI, arterial digital subtraction angiography, echocardiography, and lumbar puncture were obtained at discretion of the attending physician. Details of variables prospectively registered in the database have been previously described [12]. Medical complications (including respiratory events, urinary complications, cardiac events, vascular events, and infection) and mortality during the acute phase of the disease were assessed. Respiratory events included aspiration pneumonia, respiratory superinfection, and pulmonary embolism. Cardiac complications were arrhythmia, heart failure, and acute myocardial infarction. Vascular complications included deep venous thrombosis and peripheral arterial embolism. Causes of death were analyzed according to criteria of Silver et al. [13].

Demographic data, risk factors, clinical events, neuroimaging findings, and outcome of patients with prolonged hospital stay and those without prolonged hospital stay (length of stay $<12$ days) were compared using the analysis of variance (ANOVA) for continuous variables and the chi-square $\left(\chi^{2}\right)$ test (with Yate's correction or Bonferroni's when necessary) for categorical variables. Variables were subjected to multivariate analysis with a logistic regression procedure and forward stepwise selection if $p<0.10$ after bivariate testing. The effect of variables on prolonged hospitalization was studied in a multiple regression model based on demographic, vascular risk factors, and clinical, neuroimaging and outcome variables, in which length of stay of 12 days or more (absent $=0$, present $=1$ ) was the dependent variable. Statistical significance was set at $p<0.05$. Age was used as a continuous variable with a constant odds ratio for each year. The level of significance to remain in the model was 0.15 . The tolerance level was established as 0.0001 . The maximum likelihood approach was used to estimate weights of the logistic parameters. The odds ratio (OR) and 95\% confidence intervals (CI) were calculated from the beta coefficients and standard errors. The hypothesis that the logistic model adequately fit the data was tested by means of the goodness-of-fit $\chi^{2}$ test.

\section{Results}

Of the 3112 acute stroke patients included in the study, prolonged hospital stay was recorded in 1536 (49.4\%). Forty-three percent were men and the mean (standard deviation, SD) age was 75.3 (12.1) years. The most frequent cardiovascular risk factor was hypertension (55.5\%). Relevant clinical features were decreased consciousness (22.5\%), sensory symptoms (42.8\%), and speech disturbances (51.6\%). Intracerebral hemorrhage was present in $59.6 \%$ of patients and ischemic infarction in $47.8 \%$. Atherothrombotic, cardioembolic, and infarction of unknown cause were the most common ischemic stroke subtypes, with involvement of the middle cerebral artery in $55.3 \%$ of cases. Overall, medical complications were recorded in $56.4 \%$ of cases $(\mathrm{n}=867)$. The mean (SD) length of hospital stay was 27.7 (24.8) days. Only 151 patients $(9.8 \%)$ were symptom free at hospital discharge. A total of 12 patients died, with an in-hospital mortality rate of $12 \%$.

When data in patients with and without prolonged hospitalization were compared (Table 1), the following variables were significantly more frequent among patients with length of stay of 12 days or more: male sex, 
Table 1. Data of 3112 patients with acute stroke.

\begin{tabular}{|c|c|c|c|}
\hline \multirow{2}{*}{ Variable } & \multicolumn{2}{|c|}{ Length of hospital stay } & \multirow{2}{*}{$p$ value } \\
\hline & 12 days or more $(\mathrm{n}=1536)$ & Less than 12 days $(n=1576)$ & \\
\hline Male sex & $655(42.6)$ & $484(30.7)$ & $<0.05$ \\
\hline Patients aged $\geq 85$ years & $335(21.8)$ & $304(19.3)$ & 0.045 \\
\hline Age, years, mean (SD) & $75.3(12.1)$ & $74.9(11.5)$ & 0.078 \\
\hline \multicolumn{4}{|l|}{ Vascular risk factors } \\
\hline Hypertension & $852(55.5)$ & $896(56.9)$ & 0.229 \\
\hline Diabetes mellitus & $338(22.0)$ & $357(22.7)$ & 0.348 \\
\hline Valve heart disease & $105(6.8)$ & $84(5.3)$ & 0.046 \\
\hline Ischemic heart disease & $229(14.9)$ & $239(15.2)$ & 0.441 \\
\hline Atrial fibrillation & $479(31.2)$ & $390(24.7)$ & 0.000 \\
\hline Congestive heart failure & $84(5.5)$ & $73(4.6)$ & 0.162 \\
\hline Peripheral vascular disease & $111(7.2)$ & $126(8.0)$ & 0.230 \\
\hline Alcohol use (>80 g/day) & $41(2.7)$ & $42(2.7)$ & 0.541 \\
\hline Smoking ( $>20$ cigarettes/day) & $135(8.8)$ & $159(10.1)$ & 0.119 \\
\hline Hyperlipidemia & $224(14.6)$ & $304(19.3)$ & 0.000 \\
\hline \multicolumn{4}{|l|}{ Clinical findings } \\
\hline Progressive onset (hours) & $152(9.9)$ & $119(7.6)$ & 0.012 \\
\hline Headache & $235(15.3)$ & $210(13.3)$ & 0.064 \\
\hline Early seizures & $38(2.5)$ & $27(1.7)$ & 0.087 \\
\hline Decreased consciousness & $346(22.5)$ & $280(17.8)$ & 0.001 \\
\hline Limb weakness & $1258(81.9)$ & $1096(69.5)$ & 0.000 \\
\hline Sensory symptoms & $657(42.8)$ & $531(33.7)$ & 0.000 \\
\hline Hemianopia & $313(20.4)$ & $242(15.4)$ & 0.000 \\
\hline Speech disturbance & $792(51.6)$ & $759(48.2)$ & 0.031 \\
\hline Ataxia & $98(6.4)$ & $112(7.1)$ & 0.231 \\
\hline Cranial nerve palsy & $64(4.2)$ & $87(5.5)$ & 0.047 \\
\hline Ischemic stroke subtype & & & 0.000 \\
\hline Atherothrombotic & $405(52.6)$ & $365(47.4)$ & \\
\hline Lacunar & $226(30.8)$ & $507(69.2)$ & \\
\hline Cardioembolic & $444(58.2)$ & $319(41.8)$ & \\
\hline Unknown cause & $146(45.1)$ & $178(54.9)$ & \\
\hline Unusual cause & $72(63.2)$ & $42(36.8)$ & \\
\hline Intracerebral hemorrhage & $243(59.6)$ & $165(40.4)$ & 0.000 \\
\hline \multicolumn{4}{|l|}{ Topography } \\
\hline Frontal & 229 (14.9) & $156(9.9)$ & 0.000 \\
\hline Parietal & $418(27.2)$ & $281(17.8)$ & 0.000 \\
\hline Temporal & $452(29.4)$ & $276(17.5)$ & 0.000 \\
\hline Occipital & $160(10.4)$ & $137(8.7)$ & 0.058 \\
\hline Basal ganglia & $250(16.3)$ & $180(11.4)$ & 0.000 \\
\hline Medulla & $24(1.6)$ & $15(1.0)$ & 0.085 \\
\hline Corona radiata & $24(1.6)$ & $43(2.7)$ & 0.017 \\
\hline Middle cerebral artery involvement & $850(55.3)$ & $722(45.8)$ & 0.000 \\
\hline Posterior cerebral artery involvement & $153(10)$ & $186(11.8)$ & 0.056 \\
\hline Anterior cerebral artery involvement & $42(2.7)$ & $38(2.4)$ & 0.324 \\
\hline Cerebral venous thrombosis & $10(0.7)$ & $3(0.2)$ & 0.042 \\
\hline \multicolumn{4}{|l|}{ Outcome } \\
\hline Respiratory events & $200(13)$ & $98(6.2)$ & 0.000 \\
\hline Urinary tract infection & $222(14.5)$ & $55(3.5)$ & 0.000 \\
\hline Cardiac complications & $76(4.9)$ & $62(3.9)$ & 0.099 \\
\hline Vascular complications & $39(1.8)$ & $10(0.6)$ & 0.002 \\
\hline Infectious complications & $330(21.5)$ & $110(7.0)$ & 0.000 \\
\hline Symptom free at discharge & $151(9.8)$ & $362(23)$ & 0.000 \\
\hline In-hospital mortality & $185(12.0)$ & $277(17.6)$ & 0.000 \\
\hline Length hospital stay, days, mean (SD) & $27.7(24.8)$ & $7.8(2.9)$ & 0.000 \\
\hline
\end{tabular}

Data as numbers and percentages in parenthesis unless otherwise stated. 
age 85 years or older, valvular heart disease, atrial fibrillation, subacute onset, decreased consciousness, limb weakness, sensory symptoms, hemianopia, speech disturbances, intracerebral hemorrhage, and respiratory, urinary, cardiac, vascular and infectious complications. Also, anticoagulation $(22.3 \%$ vs $13.2 \%)$ and treatment with antibiotics $(22.9 \%$ vs $8.6 \%)$ was more frequent in patients with prolonged hospital stay.

In the multivariate analysis, factors independently associated with prolonged length of hospital stay were male sex, limb weakness, vascular complications, urinary complications, and infectious complications (Table 2). In contrast, symptom free at discharge and lacunar infarction were inversely associated with prolonged hospitalization.

\section{Discussion}

Accurate information about hospital resource utilization is necessary for management of health care services. The analysis of prolonged length of hospital stay can provide valuable data for planning and policy in the health care system. The average length of stay for stroke patients varies among different countries, and may reflect the impact of the differences in health care organization. The length of stay in USA for acute ischemic stroke ranges from 6 to 11 days [1] compared with much longer hospitalization (17 to 26 days) in Canada [14], Europe [2], and Asia [15]. In the present study, the mean length of stay for patients with prolonged hospitalization was 27.7 days, significantly longer than patients discharged from the hospital after less than 12 days of admission.

An important finding of our study was the dominant relevance of medical complications developed during in-patient care for predicting prolonged length of stay in acute stroke victims. Vascular complications, urinary infection and other infectious complications were independent factors associated with prolonged hospital stay. The presence of infections of any type or source is a cause of acute neurological deterioration, a situation that increases both mortality and morbidity in acute stroke patients [16]. It has been shown that stroke-associated infection, in particular pneumonia, is independently associated with poor functional outcome after ischemic stroke [17]. Urinary events, including urinary tract infection, incontinence, or urinary retention are the most common complication in stroke patients and may be the source of bloodstream infection. Deep venous thrombosis is the most frequent vascular complication and may be the cause of pulmonary thromboembolism, a common cause of death during the acute phase of stroke. In the presence of these complications, the need to make the diagnosis and to establish the appropriate pharmacological treatment are circumstances that extend the length of hospital stay. Also, it has been shown that length of stay is independently decreased for patients treated in hospitals providing acute stroke unit services $[3,18]$.

Other clinical predictors related to the severity of stroke, such as limb weakness or symptom free at discharge are consistent with data reported in other studies $[1,2,15,19]$. In the study of Appelros [19], items of the NIH stroke scale that best correlated with length of stay for stroke patients were paresis, unilateral neglect, and level of consciousness. Functional disability, the lack of ability to perform an activity or task in the range considered normal for an individual, is an important outcome after non-fatal stroke. More interesting, lacunar infarct was the only acute stroke subtype that was inversely associated with prolonged hospital stay. This may be explained by the small infarct size $(<2 \mathrm{~cm})$ and the favorable short-term prognosis of lacunar stroke as compared with other ischemic stroke subtypes [20].

Male sex was another independent predictor of prolonged hospital stay. Cohort studies indicate that women and men have differences in risk factors profiles, acute stroke presentation, and stroke etiology [21] but clinical outcome as well as the number of patients with a favorable clinical outcome did not differ significantly between women and men [22]. Male sex was also a predictive factor of prolonged hospitalization in the study of Chang et al. [15]. It is not clear whether the influence of sex on length of hospital stay reflects the impact of culture difference or is due to other factors.

Table 2. Independent predictive value of different variables associated with prolonged hospital stay in acute stroke patients.

\begin{tabular}{ccccc}
\hline Variable & $\beta$ & SE $(\beta)$ & Odds ratio (95\% confidence interval) & $p$ value \\
\hline Vascular complications & 0.987 & 0.344 & $2.68(1.37$ to 5.26$)$ & 0.004 \\
Urinary complications & 0.940 & 0.177 & $2.56(1.81$ to 3.62$)$ & 0.000 \\
Limb weakness & 0.584 & 0.090 & $1.79(1.50$ to 2.14$)$ & 0.000 \\
Infectious complications & 0.581 & 0.135 & $1.78(1.37$ to 2.33$)$ & 0.000 \\
Male sex & 0.154 & 0.077 & $1.16(1.01$ to 1.35$)$ & 0.045 \\
Symptom free at discharge & -0.806 & 0.109 & $0.45(0.36$ to 0.55$)$ & 0.000 \\
Lacunar infarction & -0.841 & 0.094 & $0.43(0.36$ to 0.52$)$ & 0.000 \\
\hline
\end{tabular}

$\beta=-3.91$, SE $[\beta]=0.911$, goodness-of-fit test $\chi^{2}=5.078, d f=8, p=0.749$. Area under the ROC curve $=0.692$, sensitivity $67 \%$, specificity $66 \%$, positive predictive value $63 \%$, negative predictive value $66 \%$, diagnostic accuracy $64.2 \%$. 


\section{Conclusion}

These results indicate that in-hospital medical complications (vascular, urinary, and infectious) are relevant factors influencing duration of hospitalization after acute stroke. Therefore, prevention of potentially modifiable risk factors for medical complications is an important aspect of the early management of patients with stroke.

\section{Acknowledgements}

The study was supported by a grant from FIS PI081514, Madrid, Spain. We thank Drs. Adela Vicens, Josep Maria Vives, and Guillermo Arbe for their valuable help in collecting data from the stroke registry, and Dr Marta Pulido for editing the manuscript and editorial assistance.

\section{REFERENCES}

[1] G. Saposnik, F. Webster, C. O’Callaghan and V. Hachinski, "Optimizing Discharge Planning: Clinical Predictors of Longer Stay after Recombinant Tissue Plasminogen Activator for Acute Stroke," Stroke, Vol. 36, No. 1, 2004, pp. 147-150. doi:10.1161/01.STR.0000150492.12838.66

[2] A. van Straten, J. H. P. van der Meulen, G. A. M. van den Bos and M. Limburg, "Length of Hospital Stay and Discharge Delays in Stroke Patients," Stroke, Vol. 28, No. 1, 1997, pp. 137-140. doi:10.1161/01.STR.28.1.137

[3] P. U. Heuschmann, P. L. Kolominsky-Rabas, B. Misselwitz, P. Hermanek, C. Leffmann, G. M. Von Reutern, L. Lachenmayer, H. J. Bücker-Nott and K. Berger, "Factors Influencing Duration of Hospitalization after Stroke in Germany," Deutsche Medizinische Wochenschrift, Vol. 129, No. 7, 2004, pp. 299-304. doi:10.1055/s-2004-818624

[4] N. Spratt, Y. Wang, C. Levi, K. Ng, M. Evans and J. Fisher, "A Prospective Study of Predictors of Prolonged Hospital Stay and Disability after Stroke," Journal of Clinical Neuroscience, Vol. 10, No. 6, 2003, pp. 665-669. doi:10.1016/i.jocn.2002.12.001

[5] R. Hara-Watanabe, Y. Inatomi, T. Yonehara, S. Fujioka, Y. Hashimoto, T. Hirano and M. Uchino, "Prolonged Factors of Length of Hospital Stay in Acute Ischemic Stroke," Rinsho Shinkeigaku, Vol. 45, No. 6, 2005, pp. 405-410.

[6] H. C. Lee, K. C. Chang, C. F. Lan, C. T. Hong, Y. C. Huang and M. L. Chang, "Factors Associated with Prolonged Hospital Stay for Acute Stroke in Taiwan," Acta Neurologica Taiwanica, Vol. 17, No. 1, 2008, pp. 17-25.

[7] Early Supported Discharge Trialists, "Services for Reducing Duration of Hospital Care for Acute Stroke Patients," Cochrane Database of Systematic Reviews, Vol. 18, No. 2, 2005, Article ID: CD000443.

[8] A. Arboix, C. Morcillo, L. García-Eroles, M. Oliveres, J. Massons and C. Targa, "Different Vascular Risk Factor Profiles in Ischemic Stroke Subtypes: A Study from the 'Sagrat Cor Hospital of Barcelona Stroke Registry'," Acta Neurologica Scandinavica, Vol. 102, No. 4, 2000, pp. 264-270. doi:10.1034/j.1600-0404.2000.102004264.x
[9] A. Arboix, J. Alvarez-Sabín, L. Soler, "Nomenclatura de las Enfermedades Vasculares Cerebrales," Neurologia, Vol. 13, Suppl. 1, 1998, pp. 1-10.

[10] Special Report from the National Institute of Neurological Disorders and Stroke, "Classification of Cerebrovascular Diseases. III,” Stroke, Vol. 21, No. 4, 1990, pp. 637 676. doi:10.1161/01.STR.21.4.637

[11] A. Arboix, L. García-Eroles, J. Massons, M. Oliveres and C. Targa, "Hemorrhagic Lacunar Stroke," Cerebrovascular Diseases, Vol. 10, No. 3, 2000, pp. 229-234. doi:10.1159/000016061

[12] A. Arboix, V. Cendrós, M. Besa, L. García-Eroles, M. Oliveres, C. Targa, M. Balcells, E. Comes and J. Massons, "Trends in Risk Factors, Stroke Subtypes and Outcome. Nineteen-Year Data from the Sagrat Cor Hospital of Barcelona Stroke Registry," Cerebrovascular Diseases, Vol. 26, No. 5, 2008, pp. 509-516. doi:10.1159/000155989

[13] F. L. Silver, J. W. Norris, A. J. Lewis and V. C. Hachinski, "Early Mortality Following Stroke: A Prospective Review," Stroke, Vol. 15, No. 3, 1984, pp. 492-496. doi:10.1161/01.STR.15.3.492

[14] J. V. Tu and Y. Gong, "Trends in Treatment and Outcomes for Acute Stroke Patients in Ontario, 1992-1998," Archives of Internal Medicine, Vol. 163, No. 3, 2003, pp. 293-297. doi:10.1001/archinte.163.3.293

[15] K. C. Chang, M. C. Tseng, H. H. Weng, Y. H. Lin, C. W. Liou and T. Y. Tan, "Prediction of Length of Stay of First-Ever Ischemic Stroke," Stroke, Vol. 33, No. 11, 2002, pp. 2670-2674. doi:10.1161/01.STR.0000034396.68980.39

[16] W. F. Westendop, P. J. Nederkoom, J. D. Vermeij, M. G. Dijkgraaf and D. van de Beek, "Post-Stroke Infection: A Systematic Review and Meta-Analysis," BMC Neurology, Vol. 11, 2011, p. 110. doi:10.1186/1471-2377-11-110

[17] F. H. Vermeij, W. J. Scholte op Reimer, P. De Man, R. J. Oostenbrugge, C. L. Franke, G. De Jong, P. L. de Kort and D. W. Dippel, "Stroke-Associated Infection Is an Independent Risk Factor for Poor Outcome after Acute Ischemic Stroke: Data from the Netherlands Stroke Survey," Cerebrosacular Diseases, Vol. 27, No. 5, 2009, pp. 465-471.

[18] E. Díez-Tejedor and B. Fuentes, "Acute Care in Stroke: Do Stroke Units Make the Difference?" Cerebrovascular Diseases, Vol. 11, Suppl. 1, 2001, pp. 31-39. doi:10.1159/000049123

[19] P. Appelros, "Prediction of Length of Stay for Stroke Patients," Acta Neurological Scandinavica, Vol. 116, No. 1, 2007, pp. 15-19. doi:10.1111/j.1600-0404.2006.00756.x

[20] A. Arboix, I. Padilla, L. García-Eroles, J. Massons, E. Comes and C. Targa, "Pure Motor Hemiparesis: A Clinical Study of 222 Patients," Journal of Neurology Neurosurgery and Psychiatry, Vol. 71, No. 2, 2001, pp. 239242. doi:10.1136/jnnp.71.2.239

[21] A. Arboix, M. Oliveres, L. García-Eroles, C. Maragall, J. Massons and C. Targa, "Acute Cerebrovascular Disease in Women," European Neurology, Vol. 45, No. 4, 2001, pp. 199-205. doi:10.1159/000052130

[22] A. Förster, A. Gass, R. Kern, M. E. Wolf, C. Ottomeyer, 
K. Zohsel, M. Hennerici and K. Szabo, "Gender Differences in Acute Ischemic Stroke. Etiology, Stroke Patterns and Response to Thrombolysis," Stroke, Vol. 40, No. 7,
2009 , pp. 2428-2432.

doi:10.1161/STROKEAHA.109.548750 